\title{
GREGARINES (APICOMPLEXA: EUGREGARINORIDA) PARASITIZING THE CAVE CRICKET TROGLOPHILUS (ORTHOPTERA: RAPHIDOPHORIDAE) IN THE SLOVENIAN KARST
}

\author{
GREGARINE (APICOMPLEXA: EUGREGARINORIDA), \\ KI PARAZITIRAJO V JAMSKIH KOBILICAH TROGLOPHILUS \\ (ORTHOPTERA: RAPHIDOPHORIDAE) NA SLOVENSKEM KRASU
}

\author{
Dušan DEVETAK ${ }^{1^{*}} \&$ Tone NOVAK ${ }^{1}$
}

\begin{abstract}
UDC 593.191:595.72(497.4)

Dušan Devetak \& Tone Novak: Gregarines (Apicomplexa: Eugregarinorida) parasitizing the cave cricket Troglophilus (Orthoptera: Raphidophoridae) in the Slovenian karst

The cave crickets Troglophilus neglectus Krauss, 1879 and T. cavicola (Kollar, 1833) originating from caves in Slovenia were surveyed for gregarines. Two gregarine species were identified. Gregarina troglophili (Golemansky \& Lipa, 1991) occurred in both cave cricket species, T. cavicola being reported a new host species. Besides, a new species, Gregarina bernardae sp. n., infecting T. neglectus, was identified and described. The two species often infest the same T. neglectus individuals, but can be well distinguished upon the shape and morphometric indexes of both solitary gregarines and associations. The deutomerite in solitary gamonts of $G$. troglophili is orbicular to broadly elliptoid, while in G. bernardae sp. n. it is oblong to dolioform. In associations, the primite deutomerite in G. troglophili is orbicular to broadly elliptoid, while in G. bernardae sp. n. it is shallowly oblong to oblong. In contrast to broadly obovoid to finely obdeltoid satellite deutomerite in G. troglophili, it is narrowly dolioform or dolioform to narrowly obdeltoid in G. bernardae sp. n. Both species are new to the faunal list of Slovenia, and to the list of cave-dwelling species in the country. We direct the attention to the parasite diversity of cave-dwelling animals - a promising, but understudied topic that might considerably contribute to the diversity of the subterranean fauna.
\end{abstract}

Keywords: Conoidasida, Gregarinasina, Gregarina troglophili, Gregarina bernardae sp. n., parasite diversity, syntopy.
Izvleček

UDK 593.191:595.72(497.4)

Dušan Devetak \& Tone Novak: Gregarine (Apicomplexa: Eugregarinorida), ki parazitirajo $v$ jamskih kobilicah Troglophilus (Orthoptera: Raphidophoridae) na slovenskem krasu V jamskih kobilicah Troglophilus neglectus Krauss, 1879 in T. cavicola (Kollar, 1833) sva preverjala prisotnost gregarin. Prepoznala sva dve vrsti gregarin. Gregarina troglophili Golemansky \& Lipa, 1991 je bila prisotna v obeh jamskih kobilicah; T. cavicola je njen novo ugotovljeni gostitelj. Poleg te sva v T. neglectus našla novo vrsto, Gregarina bernardae sp. n., ki jo tu opisujeva. Obe vrsti sta pogosto v istih osebkih T. neglectus, vendar se dobro razlikujeta po obliki in morfometrijskih indeksih posameznih gregarin kot asociacij. Devtomerit posameznih G. troglophili je kroglast do široko eliptičen, medtem ko je pri $G$. bernardae sp. n. podolgovat do sodčkast. V asociacijah je devtomerit primita pri G. troglophili kroglast do široko eliptičen, pri G. bernardae sp. n. pa plitvo podolgovat do podolgovat. Za razliko od široko do fino vzdolžno nesomerno jajčastega devtomerita satelita pri G. troglophili, je ta pri G. bernardae sp. n. ozko sodčkast do sodčkast ali ozko deltoidne oblike. V prispevku prikazujeva tudi sizigij pri obeh vrstah. Obenem želiva opozoriti na vrstno pestrost zajedavcev v podzemlju živečih organizmov - obetajoče, a slabo raziskano področje, ki bi lahko znatno prispevalo k povečanju diverzitete podzemeljske favne. Ključne besede: Conoidasida, Gregarinasina, Gregarina troglophili, Gregarina bernardae sp. n., raznovrstnost zajedavcev, sobivanje v habitatu.

\footnotetext{
${ }^{1}$ Department of Biology, Faculty of Natural Sciences and Mathematics, University of Maribor, Koroška cesta 160, SI-2000 Maribor, Slovenia, e-mails: dusan.devetak@guest.arnes.si; tone.novak@guest.um.si

* Corresponding author
} 


\section{INTRODUCTION}

For many reasons, karst attracts much attention among landscapes (Knez \& Kranjc, 2009). Special concerns are dedicated to economy issues, such like bauxite deposits and economically interesting elements in these deposits (Mongelli et al., 2017), water supply (Blatnik et al., 2020) and an often outstanding biodiversity above and below ground with a high degree of endemism, and markedly different species assemblages (Williams, 2008; Deharveng \& Bedos, 2018; Moldovan, 2018; Culver \& Pipan, 2019). The endemism refers to free-living cave-dwelling biota (Bole et al., 1993; Moldovan, 2012; Sket, 2012), as well as to parasites of subterranean taxa. Scarce reports on parasites for subterranean species may refer to either their actual rarity or under-sampling (Humphreys, 2018). Unfortunately, the research in parasites of cave-dwelling species is rare; in this way, we are missing important data on diversity, and several interesting issues related to parasitism in hosts specialized to living in the subterranean environment (Golemansky \& Bonnet, 1994).

Among parasites, prevalently ectoparasites, like Protozoa (Golemansky \& Bonnet, 1994), Temnocephalida (Matjašič, 1959), Acari (Dusbábek, 1998) and Diptera (Matile, 1994; Obame-Nkoghe et al., 2017), have been reported, mostly in cave-dwelling vertebrates. Among endoparasites, some Protozoa-mostly Apicomplexa and Ciliophora-(Golemansky \& Bonnet, 1994; Muzzell et al., 1997), Trematoda and Nematoda (Muzzell et al., 1997) and Acanthocephala (Amin et al., 2019) were recorded, both in vertebrates and invertebrates.

In cave-dwelling Holarctic Orthoptera and Myriapoda, at the end of the past century, Golemansky and Bonnet (1994) counted 25 species of Gregarinida, which directed them to the conclusion that the number of endoparasitic species is not lesser in subterranean hosts in comparison to the epigean hosts. However, in orthopterans this is expected, since most of them are not troglobiotic species and share both the hypogean and epigean ecophases in their life cycle (Novak \& Kuštor, 1983; Dessuter-Grandcolas et al., 1998; Lipovšek et al., 2011).
Thus, the Golemansky and Bonnet (1994) and Humphreys (2018) observations of under-sampling are still in power; a challenge for a systematic comparative study of endoparasites in cave-dwelling hosts.

Gregarines parasitizing subterranean arthropods are poorly known; indeed, only sporadic findings from cave-dwelling centipedes, millipedes and insects have been hitherto reported (e.g., Watson, 1916; Semans, 1939, 1943; Théodoridès \& Ormières, 1959; Golemansky \& Lipa, 1991; Golemansky \& Bonnet, 1994; Golemansky, 2015).

Almost a dozen of gregarine species has been described in the cave crickets of the family Rhaphidophoridae, most of them in Ellis'es and Watson's papers a hundred years ago in North America (Ellis, 1913a, 1913b; Watson, 1915, 1916; Semans, 1939, 1943; Desportes \& Schrével, 2013). At the end of the $20^{\text {th }}$ century the only European rhaphidophorid gregarine, Gregarina troglophili Golemansky \& Lipa, 1991, was described in Bulgaria, parasitizing the cave cricket Troglophilus neglectus Krauss (Golemansky \& Lipa, 1991). While early papers on the gregarines parasitizing the Rhaphidophoridae provide only rough morphological description of solitary individuals and the description of their associations, Golemansky and Lipa (1991) report metric data sufficient to characterize both trophozoites and gamonts.

During our ecological and biological research in subterranean habitats in Slovenia, we recorded, for the first time, one known, and one new gregarine species from two native cave cricket species, Troglophilus neglectus Krauss, 1879 and T. cavicola (Kollar, 1833). These two cave crickets are the most important non-troglobiotic invertebrates in central Europe with respect to their mass and energy bulk in caves (Novak et al., 2013). Here we deal with the two gregarine species; we provide an emended description of Gregarina troglophili Golemansky \& Lipa, 1991 and describe the newly-discovered species, Gregarina bernardae sp. n.

\section{MATERIAL AND METHODS}

Twenty-four adult and immature Troglophilus neglectus and three $T$. cavicola adults were taken alive manually from the following caves: Bečka jama (Cad. No. 6275, according to the Cave Register of the Karst Research Institute ZRC SAZU and Speleological Association of Slovenia; $45.66962^{\circ} \mathrm{N}, 15.34421^{\circ} \mathrm{E}, 185 \mathrm{~m}$ a.s.l.) near Metlika,
Ferranova buža (Cad. No. 8085; $45.97627^{\circ} \mathrm{N}, 14.25206^{\circ}$ E, $660 \mathrm{~m}$ a.s.l.) near Vrhnika, and Zguba jama (Cad. No. 6290; $45.79648^{\circ} \mathrm{N}, 14.21377^{\circ} \mathrm{E}, 561 \mathrm{~m}$ a.s.1.) near Postojna-all of them in the Slovenian Dinaric karst area-and transported to the Laboratory of Animal Physiology and Ethology at the University of Maribor. 
The crickets were eviscerated and the alimentary canal (diagram in Novak \& Kuštor, 1983, Fig. 5: digestive tube) dissected in insect Ringer's solution (Laughton et al., 2011). Live gregarines were observed in Ringer's solution. Permanent microscope slide preparations of gregarines were made using wet smears of the cave crickets gut tissues with parasites, and fixed by flotation on hot fixative (ethanol, formaldehyde, and acetic acid) (Clopton \& Hays, 2006). Then, the preparations were stained with Semichon's acetocarmine (Semichon, 1924), dehydrated in ethanol series, cleared in xylene series, and mounted in Canada balsam.

Gregarines were photographed using a Nikon E 800 microscope with a mounted digital camera Nikon
DN100. Thirty associations and 25 solitary gamonts of each species were measured. Measurements were taken from the digitized images of living specimens using Eclipse Net v. 1.16.3 image analysis software. Gregarine anatomical and ontogenetic nomenclature largely follows that proposed by Levine (1971), deferring to Clopton (2009) on syzygy and association. Terminology for shapes of planes and solids follows Clopton (2004).

Statistical analyses were performed in GraphPad Prism 6 software. Normality of data obtained was tested using the Shapiro-Wilk test. Given that the data tested were normally distributed, differences of means for two species were tested using the Student's $t$-test.

\section{RESULTS}

In both Troglophilus neglectus and T. cavicola, Gregarina troglophili was recorded; T. cavicola was for the first time found to be the host of this species. In addition to G. troglophili, a new Gregarina species was observed in T. neglectus only and is described here. Since the origi- nal description of the Gregarina troglophili Golemansky \& Lipa, 1991 is based on a small number of individuals (Golemansky \& Lipa, 1991), we here provide a more complete description of the species, enabled by a larger sample.
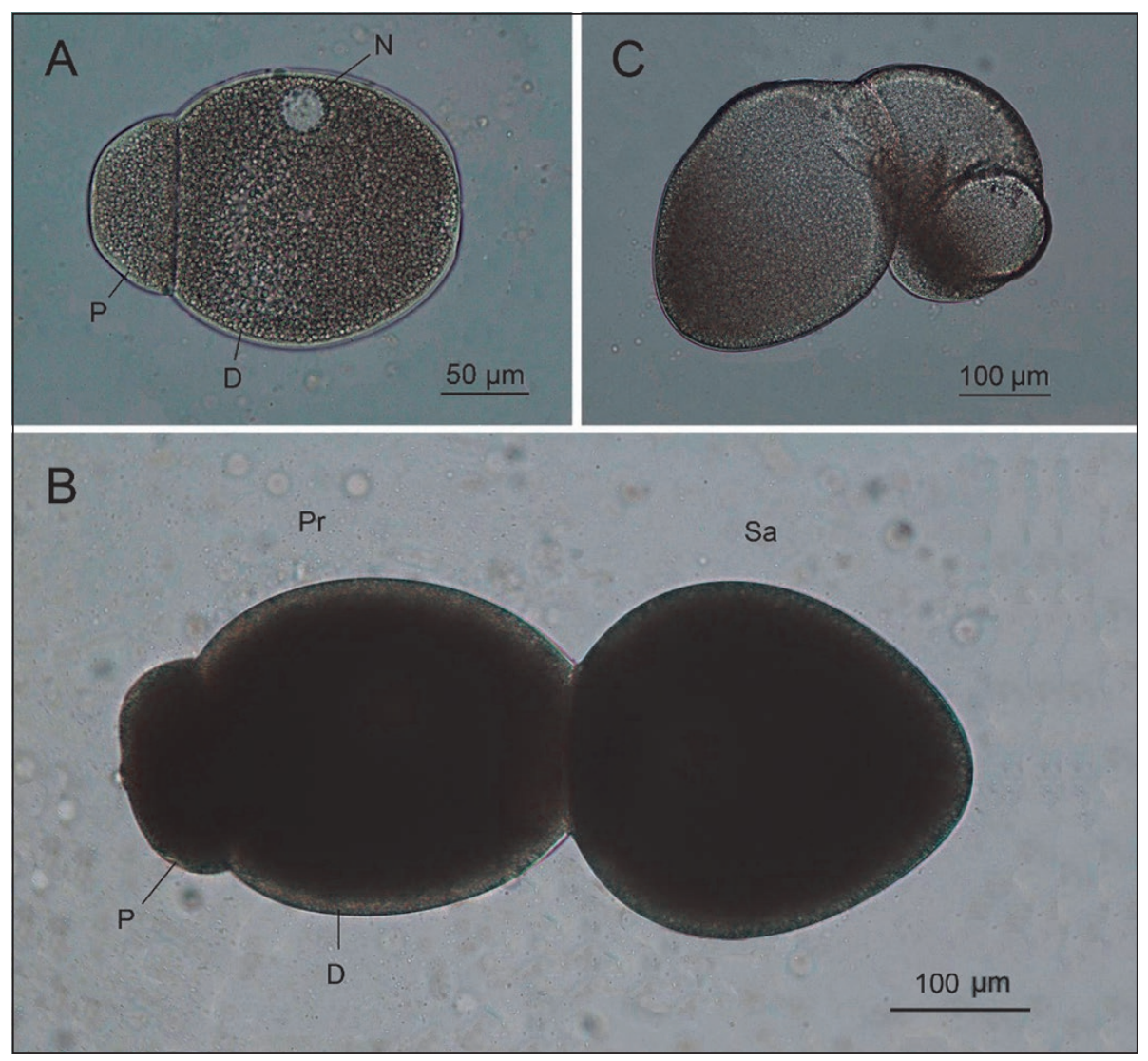

Figure 1. Live specimens of Gregarina troglophili. A) solitary gamont; B) mature gamonts in association; C) syzygy. Abbreviations: $D$ - deutomerite; $N$ - nucleus; $P$ - protomerite; $\mathrm{Pr}$ - primite; $\mathrm{Sa}$ satellite. 


\section{Gregarina troglophili Golemansky \& Lipa, 1991}

(Figures 1A-C)

\section{Material:}

Host: Troglophilus neglectus Krauss, 1879

Localities and prevalence:

- Bečka jama, 15. 6. 2012, 3/4 individuals infected (G. troglophili is co-occurring with G. bernardae sp. n.).
- Ferranova buža, 11. 6. 2012, 3/10 individuals infected; 28. 11. 2012, 3/5 individuals infected (G. troglophili is co-occurring with $G$. bernardae sp. n.).

- Zguba jama, 6. 6. 2012, 1/5 individuals infected.

Host: Troglophilus cavicola (Kollar, 1833)

Localities and prevalence:

- Ferranova buža, 11. 6. 2012, 1/3 individuals infected.

New host report.

Table 1: Morphometric data for solitary gamonts of Gregarina troglophili and G. bernardae sp. $n . N=25$ individuals. Statistically significant differences in bold. DL - deutomerite length; DWE - deutomerite width at equatorial axis; DWM - maximum deutomerite width; PL - protomerite length; PWE - protomerite width at equatorial axis; PWM-maximum protomerite width; SWT-p values of Shapiro-Wilk test; TL - total length. All measures in $\mu m$.

\begin{tabular}{|l|c|c|c|c|c|c|c|}
\hline & \multicolumn{4}{|c|}{ G. troglophili } & \multicolumn{2}{c|}{ G. bernardae sp. $\mathrm{n}}$. & t-test \\
\hline & Mean \pm SD & Range & SWT & Mean \pm SD & Range & SWT & $d f=48$ \\
\hline TL & $220.6 \pm 72.4$ & $148-396$ & 0.062 & $197.2 \pm 73.5$ & $110-311$ & 0.062 & $t=1.132 p=0.263$ \\
\hline PL & $45.9 \pm 7.4$ & $35-58$ & 0.120 & $37.2 \pm 10.2$ & $23-51$ & 0.072 & $\mathbf{t}=\mathbf{3 . 4 3 3} \mathbf{p}=\mathbf{0 . 0 0 1}$ \\
\hline DL & $174.6 \pm 67.6$ & $113-343$ & 0.055 & $160.0 \pm 63.8$ & $86-261$ & 0.051 & $t=0.790 p=0.433$ \\
\hline PWM & $104.6 \pm 38.6$ & $72-190$ & 0.062 & $61.0 \pm 23.2$ & $38-102$ & 0.077 & $\mathbf{t}=\mathbf{4 . 8 3 5} \mathbf{p}<\mathbf{0 . 0 0 1}$ \\
\hline PWE & $92.7 \pm 33.5$ & $66-165$ & 0.051 & $58.9 \pm 23$ & $34-102$ & 0.056 & $\mathbf{t}=\mathbf{4 . 1 5 7} \mathbf{p}=\mathbf{0 . 0 0 1}$ \\
\hline DWM & $159.2 \pm 71.7$ & $102-320$ & 0.071 & $79.7 \pm 31$ & $52-140$ & 0.100 & $\mathbf{t}=\mathbf{5 . 0 8 7} \mathbf{p}<\mathbf{0 . 0 0 1}$ \\
\hline DWE & $155.5 \pm 69.8$ & $100-305$ & 0.110 & $77.5 \pm 30.5$ & $51-140$ & 0.055 & $\mathbf{t}=\mathbf{5 . 1 2 0} \mathbf{p}<\mathbf{0 . 0 0 1}$ \\
\hline
\end{tabular}

Table 2: Morphometric data for gamonts in associations of Gregarina troglophili and G. bernardae sp. $n . N=30$ associations. Statistically significant differences in bold. PrDL - primite deutomerite length; PrDWE - primite deutomerite width at equatorial axis; PrDWM maximum primite deutomerite width; PrPL - primite protomerite length; PrPWE - primite protomerite width at equatorial axis; PrPWM - maximum primite protomerite width; PrTL - primite total length; SaDL - satellite deutomerite length; SaDWE - satellite deutomerite width at equatorial axis; SaDWM - maximum satellite deutomerite width; SaPL - satellite protomerite length; SaPWE - satellite protomerite width at equatorial axis; SaPWM - maximum satellite protomerite width; SaTL - satellite total length; SWT - P values of ShapiroWilk test. All measures in $\mu m$.

\begin{tabular}{|c|c|c|c|c|c|c|c|}
\hline & \multicolumn{3}{|c|}{ G. troglophili } & \multicolumn{3}{|c|}{ G. bernardae sp. n. } & \multirow{2}{*}{$\begin{array}{c}t \text {-test } \\
\mathrm{df}=58\end{array}$} \\
\hline & Mean \pm SD & Range & SWT & Mean \pm SD & Range & SWT & \\
\hline \multicolumn{8}{|l|}{ Primite } \\
\hline PrTL & $221.1 \pm 77.3$ & $92-365$ & 0.440 & $310.3 \pm 47.4$ & $228-390$ & 0.360 & $t=5.393, p<0.001$ \\
\hline PrPL & $45.9 \pm 14.8$ & $20-75$ & 0.370 & $57.5 \pm 11.1$ & $38-76$ & 0.300 & $t=3.445, p=0.001$ \\
\hline PrDL & $175.2 \pm 66.4$ & $72-295$ & 0.180 & $252.7 \pm 37.8$ & $188-326$ & 0.550 & $t=5.561, p<0.001$ \\
\hline PrPWM & $100.6 \pm 37.8$ & $50-193$ & 0.051 & $106.0 \pm 22.2$ & $70-145$ & 0.140 & $t=0.675, p=0.503$ \\
\hline PrPWE & $90.4 \pm 32.9$ & $47-173$ & 0.062 & $103.7 \pm 21.7$ & $69-145$ & 0.170 & $t=1.854, p=0.069$ \\
\hline PrDWM & $159.0 \pm 63.9$ & $68-315$ & 0.075 & $126.9 \pm 29.8$ & $79-180$ & 0.051 & $t=2.487, p=0.015$ \\
\hline PrDWE & $157.3 \pm 63.3$ & $66-312$ & 0.077 & $123.3 \pm 28.9$ & $77-180$ & 0.071 & $t=2.673, p=0.010$ \\
\hline \multicolumn{8}{|l|}{ Satellite } \\
\hline SaTL & $203.8 \pm 70.3$ & $110-300$ & 0.051 & $333.5 \pm 37.3$ & $277-408$ & 0.071 & $t=8.924, p<0.001$ \\
\hline SaPL & $21.8 \pm 5.7$ & $10-31$ & 0.260 & $30.4 \pm 8.3$ & $18-53$ & 0.053 & $t=4.673, p<0.001$ \\
\hline $\mathrm{SaDL}$ & $182 \pm 70.8$ & $100-278$ & 0.052 & $303.1 \pm 39.4$ & $242-375$ & 0.062 & $t=8.184, p<0.001$ \\
\hline SaPWM & $88.5 \pm 38.6$ & $37-160$ & 0.051 & $82.4 \pm 18$ & $58-120$ & 0.051 & $t=0.780, p=0.438$ \\
\hline SaPWE & $79.5 \pm 29.8$ & $37-126$ & 0.060 & $78.0 \pm 16.9$ & $53-110$ & 0.100 & $t=0.245, p=0.807$ \\
\hline SaDWM & $138.8 \pm 61.8$ & $50-224$ & 0.053 & $125.0 \pm 30.1$ & $87-195$ & 0.051 & $t=1.100, p=0.276$ \\
\hline SaDWE & $132.2 \pm 57.2$ & $49-216$ & 0.051 & $114.3 \pm 26.3$ & $80-183$ & 0.051 & $t=1.550, p=0.125$ \\
\hline
\end{tabular}




\section{Description:}

- Solitary gamonts (Figure 1A): Total length 148-396 $\mu \mathrm{m}$. Protomerite shallowly to broadly ovoid, markedly constricted at protomerite-deutomerite septum. Deutomerite orbicular to broadly elliptoid. Nucleus orbicular. Measurements and morphometric indexes of solitary gregarines are given in Tables 1 and 3 .

- Associations (Figure 1B): Gamonts anisomorphic; epimerite absent; associations caudo-frontal.

- Primite: Total length 92-365 $\mu \mathrm{m}$. Protomerite broadly or shallowly ovoid to shallowly elliptoid. Deutomerite orbicular to broadly elliptoid, constricted at protomerite-deutomerite septum. Nucleus orbicular. Measurements and morphometric indexes of primites are presented in Tables 2 and 4.

- Satellite: Total length 110-300 $\mu \mathrm{m}$. Protomerite depressed or very depressed ovoid to very depressed oblong. Deutomerite broadly obovoid to finely obdeltoid, not constricted at protomerite-deutomerite septum. Measurements and morphometric indexes of satellites are presented in Tables 2 and 4.

Syzygy is presented in Figure 1C.

\section{Gregarina bernardae sp. $\mathbf{n}$.}

(Figures 2-4)

\section{Material:}

Host: Troglophilus neglectus Krauss, 1879

Localities and prevalence:

- Bečka jama, 15. 6. 2012, 3/4 individuals infected (the new species is co-occurring with $G$. troglophili).

- Ferranova buža, 28. 11. 2012, 3/5 individuals infected (the new species is co-occurring with G. troglophili).

\section{Description:}

- Solitary individuals (Figures 2B, 4A): Total length 110-311 $\mu \mathrm{m}$. Protomerite shallowly ovoid to shallowly oblong, markedly constricted at protomerite-deutomerite septum. Deutomerite oblong to dolioform. Nucleus orbicular. Measurements and morphometric indexes of solitary gregarines are presented in Tables 1 and 3.

- Associations (Figures 2A, 3): Gamonts anisomorphic; epimerite absent; associations caudo-frontal.

- Primite: Total length 228-390 $\mu \mathrm{m}$. Protomerite shallowly oblong or shallowly dolioform to shallowly ovoid. Deutomerite shallowly oblong to oblong, constricted at protomerite-deutomerite septum. Nucleus orbicular. Measurements and morphometric indexes of primites are presented in Tables 2 and 4.

- Satellite: Total length 277-408 $\mu \mathrm{m}$. Protomerite shallowly or very shallowly oblong to depressed oblong. Deutomerite narrowly dolioform or dolioform to narrowly obdeltoid, constricted at protomerite-deutomerite septum. Nucleus orbicular. Measurements and morphometric indexes of satellites are presented in Tables 2 and 4.

Syzygy is presented in Figure 4B.

Oocysts of Gregarina sp.: Unfortunately, we found oocysts exclusively in the Troglophilus individuals infested with both Gregarina species. Since oocysts of $G$. troglophili have not been described, we could not identify whether the oocysts belonged to G. troglophili or G. bernardae.

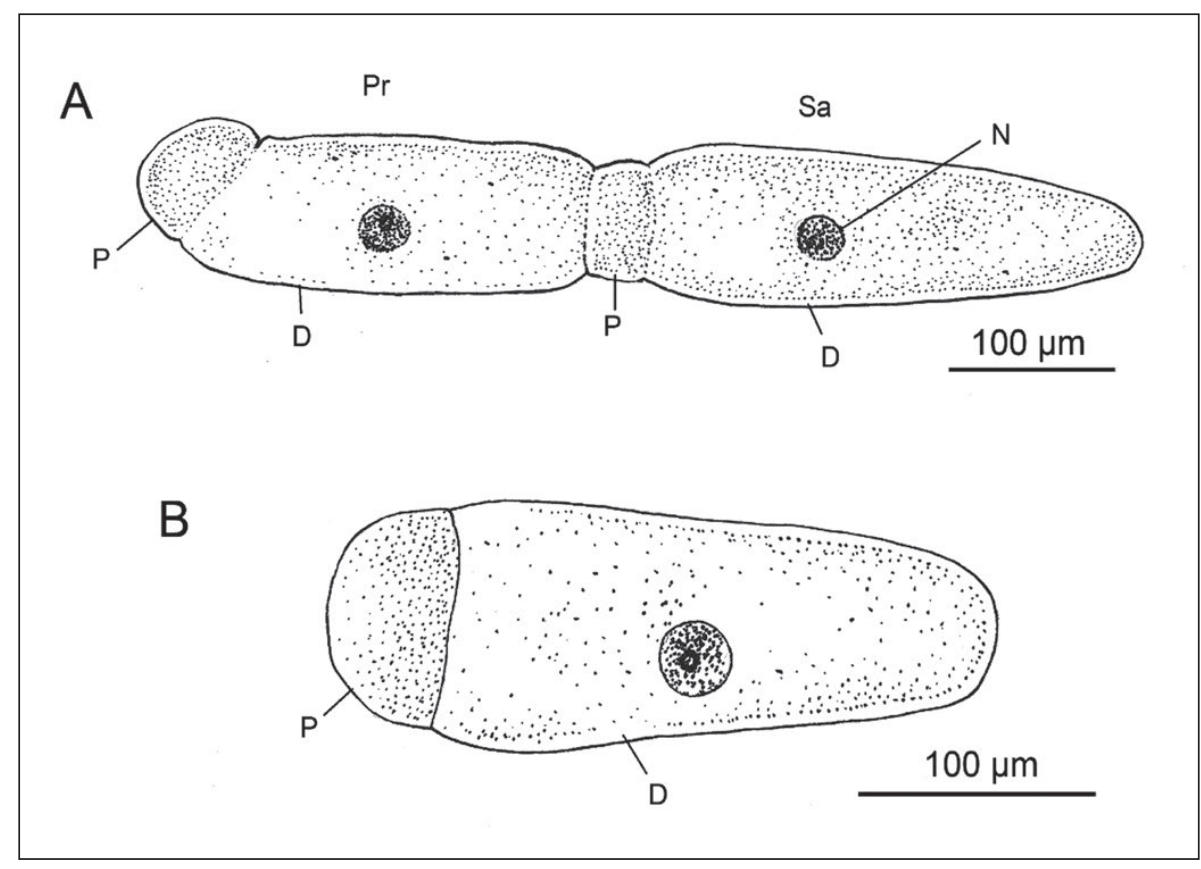

Figure 2: Specimens of Gregarina bernardae sp. n. A) mature gamonts in association; B) solitary gamont. For abbreviations see Figure 1. 


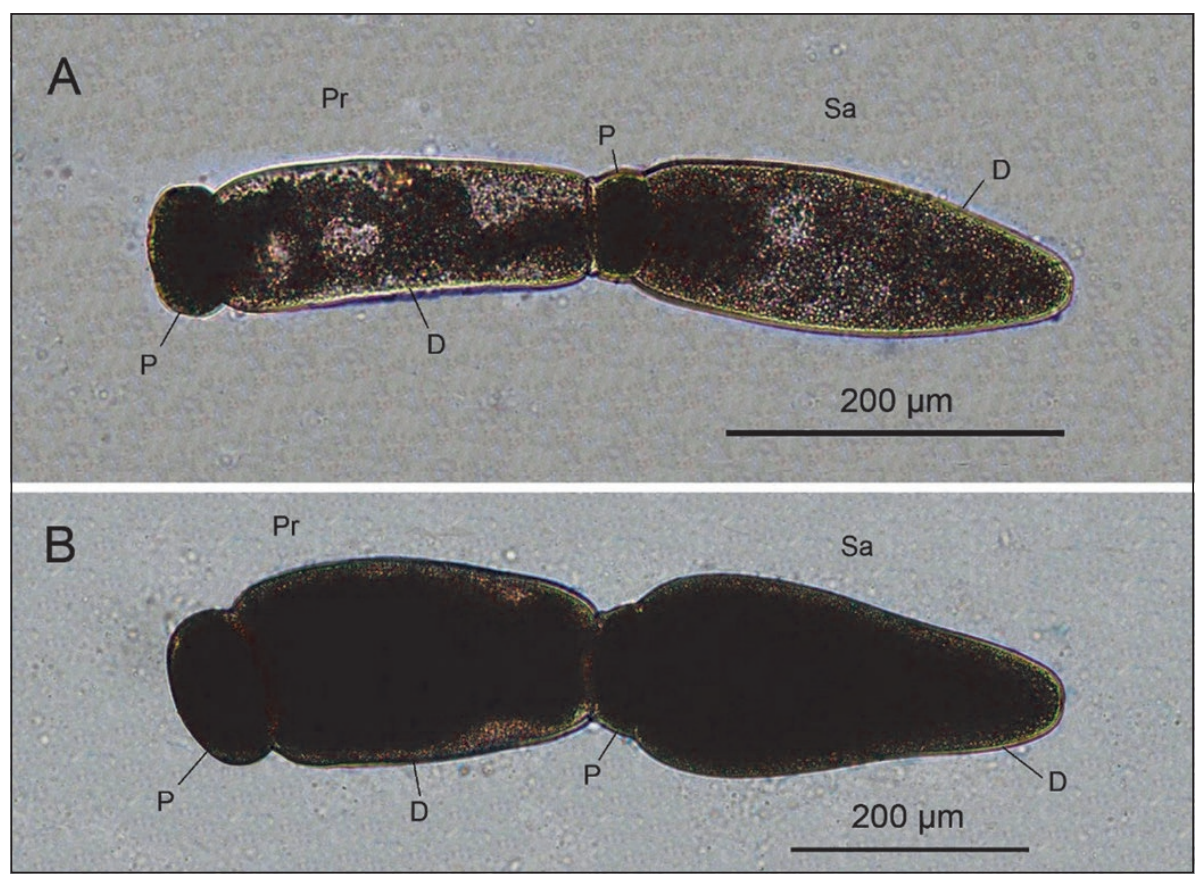

Figure 3: Live specimens of Gregarina bernardae sp. n. A, B) mature gamonts in association. For abbreviations see Figure 1.

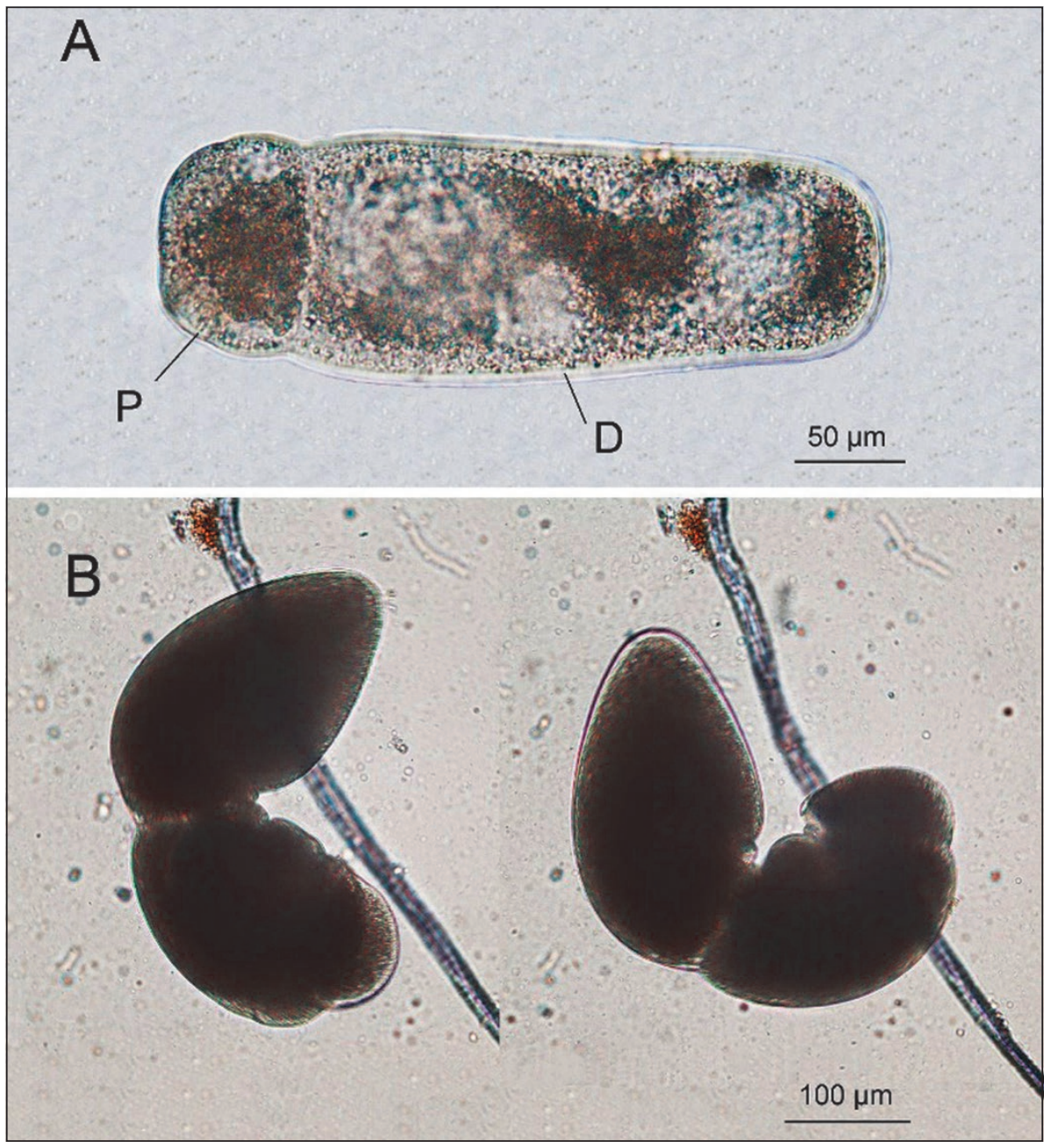

Figure 4: Live specimens of Gregarina bernardae sp. n. A) solitary gamont; B) syzygy. For abbreviations see Figure 1. 
Table 3: Morphometric indexes for solitary gamonts of Gregarina troglophili and G. bernardae sp. $n . N=25$ individuals. Statistically significant differences in bold. DL/DWM - deutomerite orbicularity; DL/PL - relative individual length; DWM/PWM - relative individual width; PL/PWM - protomerite orbicularity; TL/DL - relative deutomerite length; TL/DWM - individual orbicularity; TL/PL - relative protomerite length.

\begin{tabular}{|l|c|c|c|c|c|}
\hline & \multicolumn{2}{|c|}{ G. troglophili } & \multicolumn{2}{c|}{ G. bernardae sp. $\mathrm{n}}$. & $t$-test \\
\hline & Mean \pm SD & Range & Mean \pm SD & Range & $\mathrm{df}=48$ \\
\hline TL/PL & $4.75 \pm 1.06$ & $3.65-7.58$ & $5.18 \pm 0.66$ & $4.34-6.33$ & $\mathrm{t}=1.717, \mathrm{p}=0.092$ \\
\hline TL/DL & $1.28 \pm 0.18$ & $1.15-1.38$ & $1.24 \pm 0.04$ & $1.19-1.30$ & $\mathbf{t}=\mathbf{2 . 7 1 7}, \mathbf{p}=\mathbf{0 . 0 0 9}$ \\
\hline TL/DWM & $1,44 \pm 0.18$ & $1.13-1.73$ & $2.49 \pm 0.39$ & $1.98-3.02$ & $\mathbf{t}=\mathbf{1 2 . 2 3 0 , p < 0 . 0 0 1}$ \\
\hline PL/PWM & $0.47 \pm 0.09$ & $0.28-0.59$ & $0.64 \pm 0.13$ & $0.48-0.89$ & $\mathbf{t}=\mathbf{5 . 2 3 6 , p < 0 . 0 0 1}$ \\
\hline DL/DWM & $1.12 \pm 0.13$ & $0.92-1.36$ & $2.00 \pm 0.30$ & $1.57-2.36$ & $\mathbf{t}=\mathbf{1 3 . 1 9 0 , p < 0 . 0 0 1}$ \\
\hline DL/PL & $3.75 \pm 1.06$ & $2.65-6.58$ & $4.18 \pm 0.66$ & $3.34-5.33$ & $\mathrm{t}=1.717, \mathrm{p}=0.092$ \\
\hline DWM/PWM & $1.49 \pm 0.12$ & $1.32-1.69$ & $1.31 \pm 0.09$ & $1.13-1.41$ & $\mathbf{t}=\mathbf{6 . 1 2 7}, \mathbf{p}<\mathbf{0 . 0 0 1}$ \\
\hline
\end{tabular}

Table 4: Morphometric indexes for gamonts in associations of Gregarina troglophili and G. bernardae sp. $n . N=30$ associations. Statistically significant differences in bold. PrDL/PrDWM - primite deutomerite orbicularity; $\mathrm{PrDL} / \mathrm{PrPL}$ - relative primite length; PrDL/SaDL - relative gamont deutomerite length; $\operatorname{PrDWM} / \operatorname{Pr} P W M$ - relative primite width; PrDWM/SaDWM - relative gamont deutomerite width; PrPL/PrPWM - primite protomerite orbicularity; PrPL/SaPL - relative gamont protomerite length; PrPWM/SaPWM - relative gamont protomerite width; $\operatorname{PrTL} / \mathrm{PrDL}$ - relative primite deutomerite length; $\operatorname{PrTL} / \mathrm{PrDWM}$ - primite orbicularity; $\operatorname{PrTL} / \mathrm{PrPL}$ - relative primite protomerite length; PrTL/SaTL - relative gamont length; SaDL/SaDWM - satellite deutomerite orbicularity; SaDL/SaPL - relative satellite length; SaDWM/SaPWM - relative satellite width; SaPL/SaPWM - satellite protomerite orbicularity; SaTL/SaDL - relative satellite deutomerite length; SaTL/SaDWM - satellite orbicularity; SaTL/SaPL - relative satellite protomerite length.

\begin{tabular}{|c|c|c|c|c|c|}
\hline & \multicolumn{2}{|c|}{ G. troglophili } & \multicolumn{2}{|c|}{ G. bernardae sp. n. } & \multirow{2}{*}{$\begin{array}{c}t \text {-test } \\
\mathrm{df}=58\end{array}$} \\
\hline & Mean \pm SD & Range & Mean \pm SD & Range & \\
\hline PrTL/PrPL & $4.91 \pm 1.34$ & $3.40-9.19$ & $5.45 \pm 0.47$ & $4.67-6.25$ & $t=2.091, p=0.041$ \\
\hline PrTL/PrDL & $1.28 \pm 0.07$ & $1.12-1.42$ & $1.23 \pm 0.02$ & $1.19-1.27$ & $t=3.655, p=0.001$ \\
\hline PrTL/PrDWM & $1.42 \pm 0.14$ & $1.16-1.63$ & $2.51 \pm 0.30$ & $2.06-3.16$ & $t=17.700, p<0.001$ \\
\hline PrPL/PrPWM & $0.47 \pm 0.1$ & $0.32-0.63$ & $0.54 \pm 0.05$ & $0.48-0.66$ & $t=3.587, p=0.001$ \\
\hline PrDL/PrDWM & $1.11 \pm 0.12$ & $0.91-1.37$ & $2.04 \pm 0.27$ & $1.67-2.66$ & $t=17.100, p<0.001$ \\
\hline PrDL/PrPL & $3.91 \pm 1.34$ & $2.40-8.19$ & $4.45 \pm 0.47$ & $3.67-5.25$ & $t=2.089, p=0.041$ \\
\hline PrDWM/PrPWM & $1.57 \pm 0.17$ & $1.34-1.90$ & $1.19 \pm 0.07$ & $1.06-1.29$ & $t=10.990, p<0.001$ \\
\hline SaTL/SaPL & $10.00 \pm 4.2$ & $4.58-17.40$ & $11.80 \pm 3.83$ & $6.28-21.80$ & $t=1.746, p=0.086$ \\
\hline SaTL/SaDL & $1.14 \pm 0.07$ & $1.06-1.28$ & $1.10 \pm 0.03$ & $1.05-1.19$ & $t=2.664, p=0.010$ \\
\hline SaTL/SaDWM & $1.58 \pm 0.35$ & $1.16-2.40$ & $2.77 \pm 0.49$ & $1.69-3.47$ & $t=10.910, p<0.001$ \\
\hline SaPL/SaPWM & $0.30 \pm 0.15$ & $0.13-0.55$ & $0.39 \pm 0.13$ & $0.18-0.62$ & $t=2.389, p=0.020$ \\
\hline SaDL/SaDWM & $1.37 \pm 0.27$ & $1.08-2.02$ & $2.51 \pm 0.42$ & $1.58-3.12$ & $t=12.500, p<0.001$ \\
\hline $\mathrm{SaDL} / \mathrm{SaPL}$ & $8.95 \pm 4.21$ & $3.58-16.40$ & $10.80 \pm 3.83$ & $5.28-20.80$ & $t=1.793, p=0.078$ \\
\hline SaDWM/SaPWM & $1.55 \pm 0.2$ & $1.29-1.93$ & $1.52 \pm 0.12$ & $1.19-1.65$ & $t=0.773, p=0.442$ \\
\hline PrTL/SaTL & $1.09 \pm 0.16$ & $0.77-1.42$ & $0.93 \pm 0.08$ & $0.80-1.07$ & $t=4.959, p<0.001$ \\
\hline $\mathrm{PrPL} / \mathrm{SaPL}$ & $2.22 \pm 0.92$ & $1.05-4.21$ & $2.04 \pm 0.75$ & $1.09-3.41$ & $t=0.845, p=0.402$ \\
\hline PrDL/SaDL & $0.97 \pm 0.15$ & $0.71-1.28$ & $0.83 \pm 0.07$ & $0.75-0.97$ & $t=4.671, p<0.001$ \\
\hline PrPWM/SaPWM & $1.21 \pm 0.36$ & $0.71-1.97$ & $1.30 \pm 0.21$ & $0.95-1.63$ & $t=1.147, p=0.256$ \\
\hline PrDWM/SaDWM & $1.23 \pm 0.38$ & $0.85-2.03$ & $1.03 \pm 0.17$ & $0.69-1.30$ & $t=2.648, p=0.010$ \\
\hline
\end{tabular}




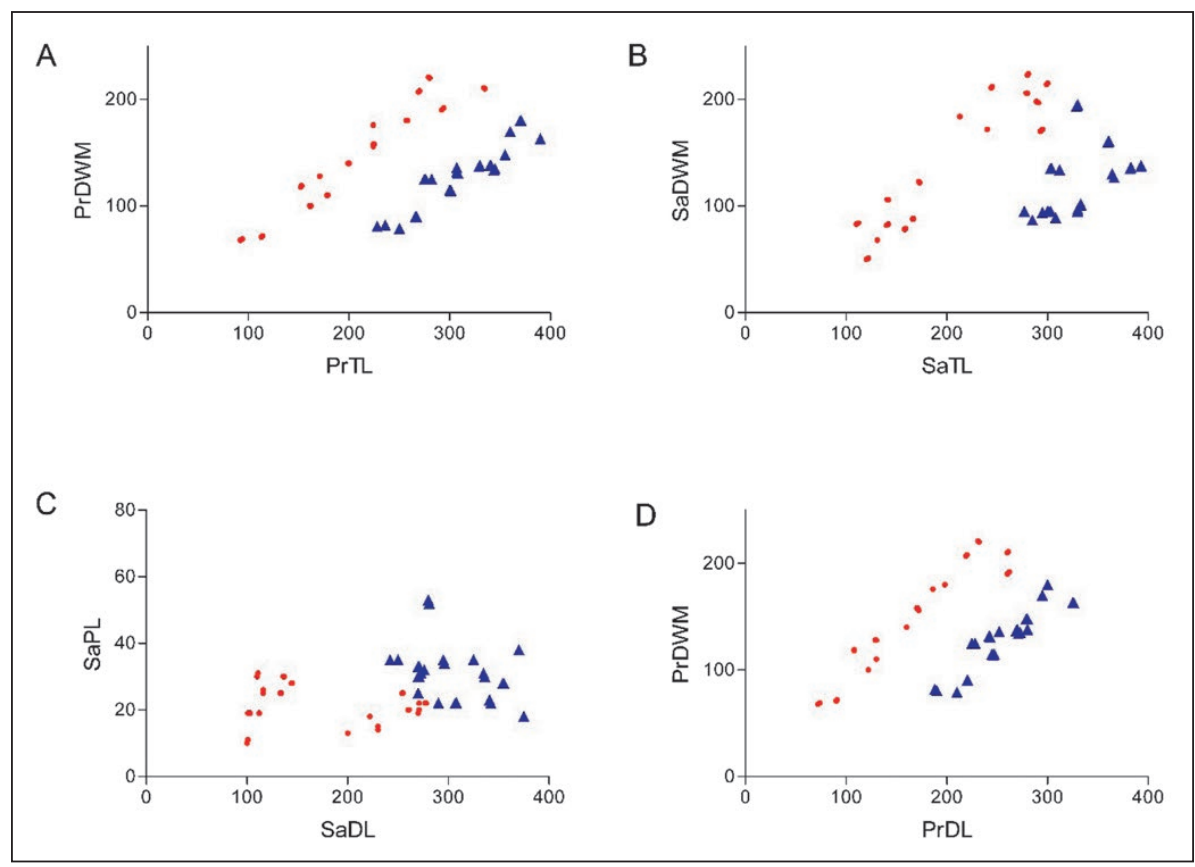

Figure 5: Morphometric indexes of associations of Gregarina troglophili and G. bernardae sp. n. A) Indexes of primite total length (PrTL) and maximum primite deutomerite width (PrDWM). B) Indexes of satellite total length (SaTL) and maximum satellite deutomerite width (SaDWM). C) Indexes of satellite deutomerite length $(\mathrm{SaDL})$ and satellite protomerite length (SaPL). D) Indexes of primite deutomerite length $(\mathrm{PrDL})$ and maximum primite deutomerite width (PrDWM). Circles - G. troglophili; triangles - G. bernardae sp. n. All measures in $\mu \mathrm{m}$.

\section{Taxonomic summary:}

Superphylum Alveolata Cavalier-Smith, 1991

Phylum Apicomplexa Levine, 1980

Class Conoidasida Levine, 1988

Subclass Gregarinasina Dufour, 1828

Order Eugregarinorida Léger, 1900

Family Gregarinidae Labbé, 1899

Genus Gregarina Dufour, 1828

Gregarina bernardae sp. n. Devetak \& Novak, 2021

- Species diagnosis: Solitary individuals with shallowly ovoid to shallowly oblong protomerite and oblong to dolioform deutomerite, on average 197.2 $\mu \mathrm{m}$ long and $79.7 \mu \mathrm{m}$ wide. Associations caudo-frontal. Shallowly oblong or shallowly dolioform to shallowly ovoid primite protomerite, on average $57.5 \mu \mathrm{m}$ long and 106 $\mu \mathrm{m}$ wide (maximum width). Shallowly oblong to oblong primite deutomerite, on average $252.7 \mu \mathrm{m}$ long and $126.9 \mu \mathrm{m}$ wide (maximum width). Shallowly or very shallowly oblong to depressed oblong satellite protomerite, on average $30.4 \mu \mathrm{m}$ long and $82.4 \mu \mathrm{m}$ wide (maximum width). Narrowly dolioform or dolioform to narrowly obdeltoid satellite deutomerite, on average $303.1 \mu \mathrm{m}$ long and $125 \mu \mathrm{m}$ wide (maximum width).

- Type host: Troglophilus neglectus Krauss, 1879 (Orthoptera: Ensifera: Rhaphidophoridae).

- Site of infection: Intestine.

- Type locality: Bečka jama, Slovenia (Cad. No. 6275; $45.66962^{\circ} \mathrm{N}, 15.34421^{\circ} \mathrm{E}, 185 \mathrm{~m}$ a.s.l., Boldraž near Metlika).

- Specimens deposited: The holotype is a hapantotype slide, deposited in the Laboratory of Animal Physiology and Ethology, FNM UM, University of Maribor, Maribor, Slovenia. The holotype is a solitary gregarine accompanied by an association on slide TROGNEG 2012-01 (Figure 2). The other solitary gregarines and associations in slides TROGNEG 2012-02 and TROGNEG 2012-03 are paratypes.

- Etymology: The specific epithet is given in honour of the first author's wife, Bernarda Devetak, who has been supporting the first author during his field and lab studies.

\section{Remarks}

Gregarina bernardae sp. $\mathrm{n}$. is clearly distinguished from G. troglophili Golemansky \& Lipa, 1991 by both morphometric indexes and size in solitary gamonts (Tables 1,3 ) and associations (Tables 2, 4). The $t$-test revealed the following significant differences in morphometric indexes of solitary individuals between the two species: relative deutomerite length, individual orbicularity (Figure 6C), protomerite orbicularity, deutomerite orbicularity (Figure 6D), and relative individual width (Table 3). Associations of Gregarina bernardae sp. n. are distinguished from those of G. troglophili by the following morphometric indexes: relative primite deutomerite length, primite orbicularity (Figure 5A), primite protomerite orbicularity, primite deutomerite orbicularity (Figure 5D), relative primite width, satellite orbicularity (Figure 5B), satellite deutomerite orbicularity (Figure 6B), relative gamont length, and relative gamont deutomerite length (Table 4). 


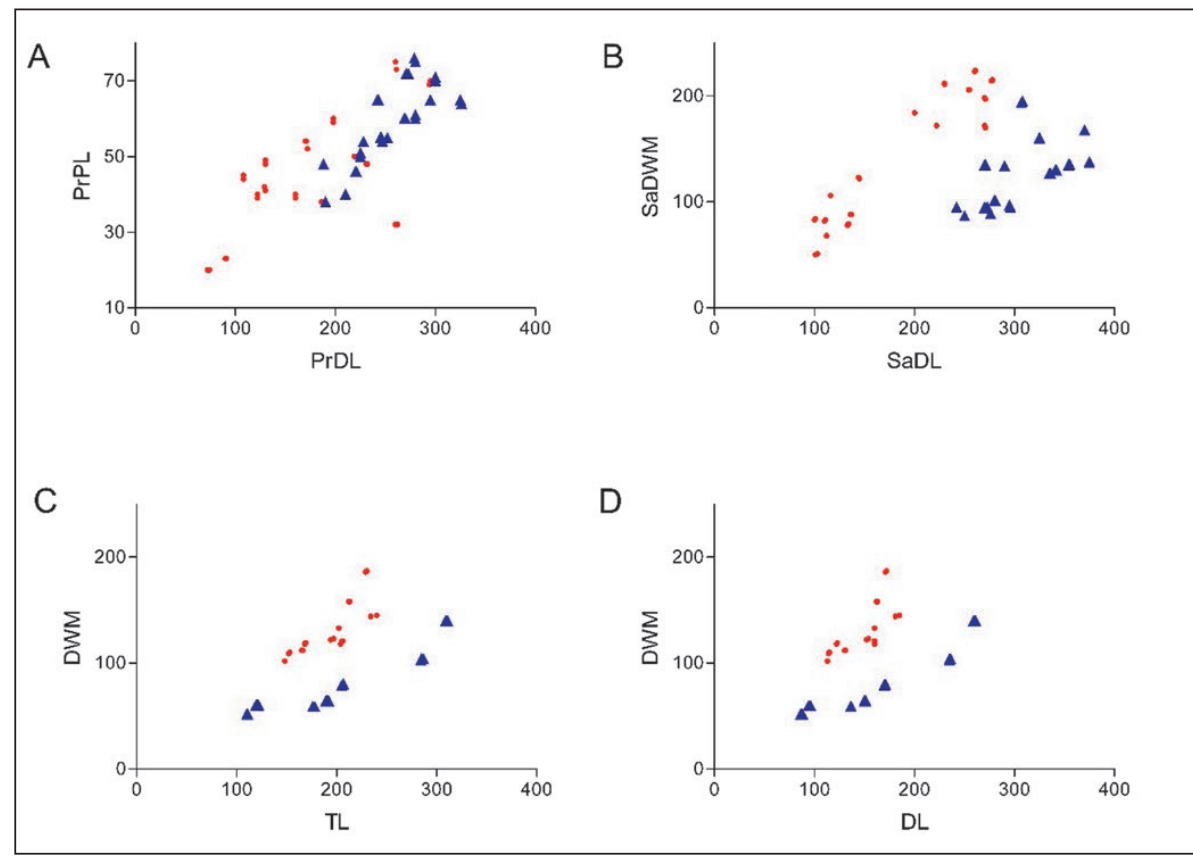

Figure 6: Morphometric indexes of associations and solitary gamonts of Gregarina troglophili and G. bernardae sp. n. A) Indexes of primite deutomerite length (PrDL) and primite protomerite length (PrPL). B) Indexes of satellite deutomerite length $(\mathrm{SaDL})$ and maximum satellite deutomerite width (SaDWM). C) Indexes of total length (TL) and maximum deutomerite width (DWM). D) Indexes of deutomerite length $(D L)$ and maximum deutomerite width (DWM). Circles - G. troglophili; triangles - G. bernardae sp. $n$. All measures in $\mu \mathrm{m}$.

\section{DISCUSSION}

Knowledge on gregarines in arthropods in Slovenia is scarce. Until now, gregarines have only been reported in some epigean insects and myriapods (Devetak et al., 2013, 2019; Devetak, 2014; Rueckert \& Devetak, 2017), but not in cave-dwelling arthropods. This paper is the first report on gregarines in the cave crickets Troglophilus neglectus and T. cavicola of the family Rhaphidophoridae from karstic caves in Slovenia. We found two species, Gregarina troglophili Golemansky \& Lipa, 1991 and G. bernardae sp. n., sometimes in the same hosts - the syntopy posing a nice opportunity for their comparison.

Our relatively rich collection of G. troglophili enabled us to provide a more comprehensive description in comparison to the original description (Golemansky \& Lipa, 1991), and a comprehensive comparison of $G$. troglophili and G. bernardae sp. n. The two species substantially differ in the shape of deutomerite. In solitary individuals, the shape of the deutomerite in G. troglophili is orbicular to broadly elliptoid, whilst in G. bernardae sp. $\mathrm{n}$. it is oblong to dolioform. In associations, the primite deutomerite in G. troglophili is orbicular to broadly elliptoid, whilst in G. bernardae sp. $\mathrm{n}$. it is shallowly oblong to oblong. In contrast to a broadly obovoid to finely obdeltoid satellite deutomerite in G. troglophili, it is narrowly dolioform or dolioform to narrowly obdeltoid in G. bernardae sp. n.

All the gregarines parasitizing in North American rhaphidophorids (Ellis, 1913a, 1913b; Watson, 1915, 1916; Semans, 1939) differ from G. troglophili by both the shape of solitary individuals and associations, but two of them, namely Gregarina ceutophili Semans, 1939 and G. hadenoeci Semans, 1939 resemble G. bernardae sp. n. In contrast to G. bernardae sp. n., the primite protomerite in G. ceutophili has sometimes blunt apex and satellite septum is devoid of a marked constriction (Semans, 1939) which is constantly present in G. bernardae sp. n. associations.

When taking into account morphometric data from the Semans' original description (Semans, 1939), G. hadenoeci significantly differs from G. bernardae sp. n. in morphometric indexes. Differences refer to the primite orbicularity (PrTL/PrDWM; G. hadenoeci: 2.43 vs G. bernardae sp. n.: 2.51), primite protomerite orbicularity (PrPL/PrPWM; 0.68 vs 0.54), primite deutomerite orbicularity (PrDL/PrDWM; 1.87 vs 2.04), satellite orbicularity (SaTL/SaDWM; 1.91 vs 2.77 ), satellite protomerite orbicularity (SaPL/SaPWM; 0.5 vs 0.39 ), and satellite deutomerite orbicularity (SaDL/SaDWM; 1.57 vs 2.51). In addition to these differences, gregarines are regarded highly host-specific parasites, generally restricted to a single host species (Levine, 1988). We did not perform any molecular phylogenetic analyses.

In conclusion, two gregarines reported here strictly differ from gregarines in other rhaphidophorid cave crickets. Although numerous papers have been published on gregarine species in Europe (see Desportes \& Schrével, 2013) and there are several monographs (e.g., Lipa, 1967; Geus, 1969), our knowledge on the diversity 
and distribution of gregarines infesting the European subterranean taxa is still deficient. The finding of a new species suggests the existence of a variety of gregarines in cave-dwelling arthropods that have not been investigated with this regard. We would like to stress that the parasite diversity of cave-dwelling animals contributes to the whole diversity of the subterranean fauna; thus, it would be worth to devote our attention into this research.

\section{ACKNOWLEDGEMENT}

We would like to thank Andrea Bardůnek Valigurová (Brno, Czech Republic), Paweł Sienkiewicz (Poznań, Poland) and an anonymous reviewer for their insightful comments on an early version of the paper. This study was supported by the Slovenian Research Agency and the Research Programme Computationally Intensive Complex Systems (P1-0403).

\section{REFERENCES}

Amin, O.M., Heckmann, R.A., Fišer, Ž., Zakšek, V., Herlyn, H., Kostanjšek, R., 2019. Description of Acanthocephalus anguillae balkanicus subsp. n. (Acanthocephala: Echinorhynchidae) from Proteus anguinus Laurenti (Amphibia: Proteidae) and the cave ecomorph of Asellus aquaticus (Crustacea: Asellidae) in Slovenia. Folia Parasitologica, 66: 015. https://doi.org/10.14411/fp.2019.015

Blatnik, M., Culver, D.C., Gabrovšek, F., Knez, M., Kogovšek, B., Kogovšek, J., Liu, H., Mayaud, C., Mihevc, A., Mulec, J., Năpăruş-Aljančič, M., Otoničar, B., Petrič, M., Pipan, T., Prelovšek, M., Ravbar, N., Shaw, T., Slabe, T., Šebela, S., Zupan Hajna, N., 2020. Water Quality Monitoring in Karst. In: Knez, M., Otoničar, B., Petrič, M., Pipan, T., Slabe, T. (Eds.), Karstology in the Classical Karst. Springer Nature Switzerland, Cham, pp. 127-137. https://doi. org/10.1007/978-3-030-26827-5

Bole, J., Drovenik, B., Mršić, N., Sket, B., 1993. Endemic animals in hypogean habitats in Slovenia. Naše jame, 35(1): 43-55.

Clopton, R.E., 2004. Standard nomenclature and metrics of plane shapes for use in gregarine taxonomy. Comparative Parasitology, 71(2): 130-140. https:// doi.org/10.1654/4151

Clopton, R.E., 2009. Phylogenetic relationships, evolution, and systematic revision of the Septate Gregarines (Apicomplexa: Eugregarinorida: Septatorina). Comparative Parasitology, 76(2): 167-190. https:// doi.org/10.1654/4388.1

Clopton, R.E., Hays, J.J., 2006. Revision of the genus Protomagalhaensia and description of Protomag- alhaensia wolfi n. comb. (Apicomplexa: Eugregarinida: Hirmocystidae) and Leidyana haasi n. comb. (Apicomplexa: Eugregarinida: Leidyanidae) parasitizing the lobster cockroach, Nauphoeta cinerea (Dictyoptera: Blaberidae). Comparative Parasitology, 73(2): 137-156. https://doi.org/10.1654/4241.1

Culver, D.C., Pipan, T., 2019. The Biology of Caves and Other Subterranean Habitats. Oxford University Press, $2^{\text {nd }}$ Ed., Oxford, U.K., pp. 301.

Deharveng, L., Bedos, A., 2018. Diversity of Terrestrial Invertebrates in Subterranean Habitats. In: Moldovan, O.T., Kováč, L., Halse, S. (Eds.), Cave Ecology. Springer Nature Switzerland, Cham, pp. 107-172. http://dx.doi.org/10.1007/978-3-319-98852-8_7

Desportes, I., Schrével, J., 2013. The Gregarines (2 vols): The early branching Apicomplexa. Treatise on Zoology - Anatomy, Taxonomy, Biology. Brill, Leiden, Boston, pp. 781.

Dessuter-Grandcolas, L., Di Russo, C., Sbordoni, V., 1998. Orthoptera. In: Juberthie, C., Decou, V. (Eds.), Encyclopaedia Biospeologica, Tome I. Société de Biospéologie, Bucarest, pp. 977-1001.

Devetak, D., 2014. Host diversity and seasonality of Hyalospora hemerobii (Apicomplexa: Eugregarinorida: Hirmocystidae) infections in lacewings. Biologia, 69(11): 1585-1592. https://doi.org/10.2478/s11756014-0456-7

Devetak, D., Omerzu, M., Clopton, R.E., 2013. Notes on the gregarines (Protozoa: Apicomplexa: Eugregarinorida) of insects in Slovenia. Annales Series Historia Naturalis 23(1): 73-89.

Devetak, D., Mihelak, K., Kos, I., 2019. Gregarines (Api- 
complexa: Eugregarinida) of Chilopoda and Diplopoda in Slovenia. Acta Zoologica Bulgarica, 71(1): 121-128.

Dusbábek, F., 1998. Acari parasiti. In: Juberthie, C., Decou, V. (Eds.), Encyclopaedia Biospeologica, Tome II. Société de Biospéologie, Bucarest, pp. 921-928.

Ellis, M.M., 1913a. Gregarines from some Michigan Orthoptera. Zoologischer Anzeiger, 43: 78-84.

Ellis, M.M., 1913b: A descriptive list of the Cephaline gregarines of the New World. Transactions of the American Microscopical Society, 32: 259-296.

Geus, A., 1969. Sporentierchen, Sporozoa: die Gregarinida der land- und süßwasserbewohnenden Arthropoden Mitteleuropas. In: Dahl, F. (Ed.), Die Tierwelt Deutschlands und der angrenzenden Meeresteile nach ihren Merkmalen und nach ihrer Lebensweise. Gustav Fischer Verlag, Jena, pp. 1-608.

Golemansky, V., 2015. Checklist of Gregarines (Apicomplexa: Eugregarinorida and Neogregarinorida) from Bulgaria. Acta Zoologica Bulgarica, 67(2): 149-157.

Golemansky, V., Bonnet, L., 1994. Protozoa. In: Juberthie, C., Decu, V. (Eds.), Encyclopaedia biospeologica I. Société de Biospeologie, Moulis and Bucarest, pp. 23-33.

Golemansky, V., Lipa, J.J., 1991. Gregarines (Apicomplexa: Eugregarinida) from cave and terrestrial arthropods in Bulgaria. Acta Protozoologica, 30: 201208.

Humphreys, W.F., 2018. Where angels fear to tread: Developments in cave ecology. In: Moldovan, O.T., Kováč, L., Halse, S. (Eds.) 2018: Cave Ecology. Springer Nature Switzerland, Cham, pp. 497-532. http://dx.doi.org/10.1007/978-3-319-98852-8_24

Knez, M., Kranjc, A., 2009. Kras. In: Pleničar, M., Ogorelec, B., Novak, M. (Eds.), The Geology of Slovenia. Geološki zavod Slovenije, Ljubljana, pp. 561-574.

Laughton, A.M., Boots, M., Siva-Jothy, M.T., 2011. The ontogeny of immunity in the honey bee, Apis mellifera L. following an immune challenge. Journal of Insect Physiology, 57(7): 1023-1032. https://doi. org/10.1016/j.jinsphys.2011.04.020

Levine, N.D., 1971. Uniform terminology for the protozoan phylum Apicomplexa. Journal of Protozoology, 18(2): 352-355. https://doi. org/10.1111/j.1550-7408.1971.tb03330.x

Levine, N.D., 1988. The phylum Apicomplexa. Volume I. CRC Press, Boca Raton, 203 pp.

Lipa, J.J., 1967. Studies on gregarines (Gregarinomorpha) of arthropods in Poland. Acta Protozoologica, 5: 97179.

Lipovšek, S., Novak, T., Janžekovič, F., Pabst, M.A., 2011. Role of the fat body in the cave crickets Troglophilus cavicola and Troglophilus neglectus (Rhaphidophor- idae, Saltatoria) during overwintering. Arthropod Structure and Development, 40(1): 54-63. https:// doi.org/10.1016/j.asd.2010.09.002

Matile, L., 1994. Diptera. In: Juberthie, C., Decou, V. (Eds), Encyclopaedia Biospeologica, Tome I. Société de Biospéologie, Bucarest, pp. 341-357.

Matjašič, J., 1959. Morfologija, biologija in zoogeografija evropskih temnocefalov in njihov sistematski položaj. Morphologie, biologie et zoogéographie des Temnocéphalides europeéne et leur situation systématique.). In : Mirković, I. (Ed.), Dissertationes SAZU, 5. Slovenska akademija znanosti in umetnosti, Ljubljana, pp. 141-181+V.

Moldovan, O.T., 2012. Beetles. In: Culver, D.C., White, W.B. (Eds.), Encyclopedia of caves. Elsevier, Amsterdam, pp. 45-51.

Moldovan, O.T, 2018. An Overview on the aquatic cave fauna. In: Moldovan, O.T., Kováč, L., Halse, S. (Eds.), Cave Ecology. Springer Nature Switzerland, Cham, pp. 173-194.

Mongelli, G., Boni, M., Oggiano, G., Mameli, P., Sinisi, R., Buccione, R., Mondillo, N., 2017. Critical metals distribution in Tethyan karst bauxite: The cretaceous Italian ores. Ore Geology Reviews, 86: 526-536. http://dx.doi.org/10.1016/j.oregeorev.2017.03.017

Muzzell, P.M., Peebles C.R., Burton, T.M., 1997. Endoparasites of plethodontid salamanders from Paradise Brook, New Hampshire. Journal of Parasitology, 83(6): 1193-1195.

Novak, T., Kuštor, V., 1983. On Troglophilus (Rhaphidophoridae, Saltatoria) from North Slovenia (YU). Mémoires de Biospéologie, 10: 127-137.

Novak, T., Janžekovič, F., Lipovšek, S., 2013. Contribution of non-troglobiotic terrestrial invertebrates to carbon input in hypogean habitats. Acta Carsologica, 42(2-3): 301-309. http://ojs.zrc-sazu.si/carsologica/article/view/669

Obame-Nkoghe, J., Leroy, E.M., Paupy, C., 2017. Diversity and role of cave-dwelling hematophagous insects in pathogen transmission in the Afrotropical region. Emerging Microbes and Infections, 6: e20. http://dx.doi.org/10.1038/emi.2017.6

Rueckert, S., Devetak, D., 2017. Gregarines (Apicomplexa, Gregarinasina) in psocids (Insecta, Psocoptera) including a new species description and their potential use as pest control agents. European Journal of Protistology, 60: 60-67. http://dx.doi. org/10.1016/j.ejop.2017.05.007

Semans, F.M., 1939. Protozoan parasites of the Orthoptera, with special reference to those of Ohio. II. Description of the Protozoan parasites recognized in this study. Ohio Journal of Science, 39: 157-181.

Semans, F.M., 1943. Protozoan parasites of the Orthop- 
tera, with special reference to those of Ohio. IV. Classified list of the Protozoan parasites of the Orthoptera of the World. Classes Mastigophora, Sarcodina and Sporozoa. Ohio Journal of Science, 43 : 221-276.

Semichon, L., 1924. Procédeé de coloration et de la regonflement des parasites animaux. Revue de Pathologie Végétale et d'Entomologie Agricole, 11: 193195.

Sket, B., 2012. Dinaric karst, diversity in. In: Culver, D.C., White, W.B. (Eds.), Encyclopedia of caves. Elsevier, Amsterdam, pp. 158-165.

Théodoridès, J., Ormières, R., 1959. Quelques eugré- garines parasites d'Arthropodes de la région de Banyuls. Vie et Milieu, 9(3): 301-324.

Watson, M.E., 1915. Some new gregarine parasites from Arthropoda. Journal of Parasitology, 2: 27-38.

Watson, M.E., 1916. Studies on Gregarines. Including descriptions of twenty-one new species and a Synopsis of the Eugregarine records from the Myriapoda, Coleoptera and Orthoptera of the World. Illinois Biological Monographs, 2: 1-258.

Williams, P., 2008. World heritage Caves and karst. IUCN Programme on Protected Areas, Gland, pp. $34+\mathrm{XVI}$. 